\title{
A variational formulation for discrete registration
}

\author{
Karteek Popuri, Dana Cobzas and Martin Jägersand \\ Department of Computing Science, University of Alberta, Edmonton, Canada
}

\begin{abstract}
We present a novel variational formulation of discrete deformable registration as the minimization of a convex energy functional that involves diffusion regularization. We show that a finite difference solution (FD) of the variational formulation is equivalent to a continuous-valued Gaussian Markov random field (MRF) energy minimization formulation previously proposed as the random walker deformable registration method [1]. A computationally efficient solution using the finite element method (FEM) method has been proposed to solve the variational minimization problem. Our proposed method obtained competitive results when compared with 14 other deformable registration methods on the CUMC12 MRI dataset.
\end{abstract}

\section{Introduction}

The task of non-rigid or deformable image registration refers to the process of finding meaningful dense correspondences between two images. It is required in a wide variety of medical imaging applications such as longitudinal studies on disease progression, multi-modal image fusion, statistical studies on anatomical variability and atlas-based segmentation. More formally, the problem of deformable image registration is posed as the estimation of an optimal displacement field that maps points in an image to the corresponding points in another image such that a similarity criterion is minimized. This minimization is inherently ill-posed due to the large number of degrees of freedom (DOF) involved and therefore it relies on regularization or smoothing constraints.

Over the years, numerous regularization approaches have been proposed for deformable registration (see [2] for a detailed review). These approaches can be broadly categorized into two groups, namely non-parametric and parametric approaches. In nonparametric approaches, explicit regularization terms such as elastic [3], diffusion [4] and curvature [5] are added to the data term and a variational minimization of the combined energy functional is performed. Alternatively, a demons minimization strategy can be used, where the smoothing of the displacement field is decoupled from the minimization of the data term [6], [7], [8]. In parametric approaches, regularization is enforced in an implicit manner through the parametrization of the displacement field using a finite set of basis functions, such as radial basis functions (RBF) [9], B-spline based free form deformations (FFD) [10], finite element method (FEM) basis functions [11].

The various non-parametric and parametric deformable registration approaches mentioned above attempt to determine the unknown displacement field through the continuous optimization of energy functionals. As an alternative to continuous formulations, there has been some interest in formulating the deformable registration task as a discrete labeling problem. Here, the space of possible displacements is discretized and 
a Markov random field (MRF) energy corresponding to the registration objective is minimized. However, as the MRF energy minimization is in general NP-hard, existing methods on MRF-based discrete deformable registration are only able to find good quality approximate solutions using graph cuts [12] and linear programming [13].

Previous discrete formulations of deformable image registration were inherently discrete in both the image domain and displacement space. In this work, we seek a formulation that keeps the image domain continuous resulting in a variational formulation for discrete deformable registration. Our formulation results in a convex functional which can be conveniently minimized to obtain a unique solution. Specifically, we associate a continuous prior probability map to each of the possible displacement values using image similarities. The prior probability maps denote how likely the corresponding displacement value is at particular spatial location in the continuous image domain. The discrete deformable registration task is then posed as a variational problem corresponding to the diffusion-based smoothing of prior probability maps. For solving this variational problem, we propose a finite element method (FEM) that employs a nonuniform mesh well adapted to the salient image features. This significantly reduces the number of DOFs involved in the minimization compared to a traditional finite difference (FD) discretization and hence leads to a highly computationally efficient solution. Further, we show that a FD discretization of our variational formulation is equivalent to the the random walker (RW) solution of discrete registration recently proposed by Cobzas et al. [1].

To summarize, we make the following contributions:

(1) We develop a novel variational formulation of discrete deformable registration on the continuous image domain. This formulation results in a convex energy functional that involves diffusion regularization.

(2) We show that a finite difference solution (FD) of the variational formulation is equivalent to a continuous-valued Gaussian MRF energy minimization formulation.

(3) We propose a computationally efficient FEM solution.

(4) We validate our method on the publicly available CUMC12 MRI dataset [14] and show a competitive performance of our proposed method in comparison to 14 other deformable registration methods.

\section{Methods}

\subsection{Variational formulation of discrete deformable registration}

Given a source image $I_{S}: \Omega_{S} \rightarrow \mathbb{R}, \Omega_{S} \subset \mathbb{R}^{\nu}$ and a target image $I_{T}: \Omega_{T} \rightarrow \mathbb{R}$, $\Omega_{T} \subset \mathbb{R}^{\nu}, \nu=2$ or 3 , the goal of non-rigid or deformable registration is to estimate a displacement field $\mathbf{U}: \Omega_{T} \rightarrow \mathbb{R}^{\nu}$ such that the warped source image $I_{S}(\mathbf{x}+\mathbf{U}(\mathbf{x}))$ is similar to the target image $I_{T}$. A similarity map $\Psi: \Omega_{T} \rightarrow \mathbb{R}$ can be defined to measure the similarity between the warped source and target images as:

$$
\Psi\left(\mathbf{x} ; \mathbf{U}, I_{T}, I_{S}\right)=\left(I_{S}(\mathbf{x}+\mathbf{U}(\mathbf{x}))-I_{T}(\mathbf{x})\right)^{2},
$$

In discrete deformable registration, the space of possible displacement values is discretized or quantized such that it corresponds to a finite set of $K$ vectors, i.e., $\mathbf{U}$ : 
$\Omega_{T} \rightarrow \mathcal{D}$, where $\mathcal{D}=\left\{\mathbf{d}^{k}\right\}_{k=1}^{K}, \mathbf{d}^{k} \in \mathbb{R}^{\nu}$. Based on the similarity map, similar to [1] we can define a prior probability map $\lambda^{k}: \Omega_{T} \rightarrow[01]$ for each of the displacement values $\mathbf{d}^{k} \in \mathcal{D}$ as:

$$
\lambda^{k}\left(\mathbf{x} ; \mathbf{d}^{k}\right)=\frac{\exp \left(-\alpha \Psi\left(\mathbf{x} ; \mathbf{d}^{k}, I_{T}, I_{S}\right)\right)}{\sum_{r=1}^{K} \exp \left(-\alpha \Psi\left(\mathbf{x} ; \mathbf{d}^{r}, I_{T}, I_{S}\right)\right)},
$$

It can be seen that the prior $\lambda^{k}\left(\mathbf{x} ; \mathbf{d}^{k}\right)$ essentially encodes the belief that the corresponding displacement value $\mathbf{d}^{k}$ is more likely at a spatial location $\mathbf{x} \in \Omega_{T}$, when the similarity measure between the warped source image and the target image at $\mathbf{x} \in \Omega_{T}$ is small. The discrete deformable registration task is next formulated as a variational energy minimization problem that estimates an unknown probability map $L^{k}: \Omega_{T} \rightarrow \mathbb{R}$ corresponding to each of the displacement values $\mathbf{d}^{k} \in \mathcal{D}$ :

$$
\begin{gathered}
\mathbf{L}^{*}=\underset{\mathbf{L}}{\operatorname{argmin}} E_{D}\left[\mathbf{L} ; I_{T}, I_{S}, \mathcal{D}\right]+\beta E_{R}^{\text {diff }}[\mathbf{L}], \\
\text { with } \quad E_{D}\left[\mathbf{L} ; I_{T}, I_{S}, \mathcal{D}\right]=\int_{\Omega_{T}}\|\mathbf{L}(\mathbf{x})-\boldsymbol{\lambda}(\mathbf{x} ; \mathcal{D})\|^{2} d \mathbf{x}, \\
\\
E_{R}^{\text {diff }}[\mathbf{L}]=\sum_{k=1}^{K} \int_{\Omega_{T}} \nabla L^{k \mathrm{~T}}(\mathbf{x}) W(\mathbf{x}) \nabla L^{k}(\mathbf{x}) d \mathbf{x},
\end{gathered}
$$

where $\boldsymbol{\lambda}=\left[\lambda^{k}\right]_{k=1}^{K}, \mathbf{L}=\left[L^{k}\right]_{k=1}^{K}, \mathbf{L}^{*}=\left[L^{* k}\right]_{k=1}^{K}$ and $\beta$ is a regularization constant. The above energy minimization formulation essentially corresponds to the anisotropic diffusion-based smoothing of the prior probability maps $\left\{\lambda^{k}\right\}_{k=1}^{K}$. In other words, the minimization attempts to find a smooth probability map $L^{k}$ associated with the displacement value $\mathbf{d}^{k}$ such that it is as "close" as possible to the corresponding prior probability map $\lambda^{k}$. The smoothness is enforced through the anisotropic diffusion-based regularization term $E_{R}^{\text {diff }}$, where $W$ is a $\nu \times \nu$ symmetric matrix denoting the diffusivity or the stiffness field. Note that in the above we do not need an explicit constraint to make sure that the unknown probability maps $\left\{L^{k}\right\}_{k=1}^{K}$ sum to 1 . This because the unique minimizer of (3) should naturally satisfy this constraint, as the prior probability maps $\left\{\lambda^{k}\right\}_{k=1}^{K}$ were defined such that they sum to 1 (like in Grady [15]). The estimated displacement field $\mathbf{U}(\mathbf{x})$ at a spatial location $\mathbf{x} \in \Omega_{T}$ is obtained by choosing the displacement value $\mathbf{d}^{k}$ with the highest optimal probability at that spatial location:

$$
\begin{gathered}
\mathbf{U}(\mathbf{x})=\mathbf{d}^{k} \\
\text { where } \quad k=\underset{r \in\{1,2, \ldots, K\}}{\operatorname{argmax}} L^{* r}(\mathbf{x}) .
\end{gathered}
$$

However, it should be noted that even though the above displacement field is constructed from the optimal probability maps $\left\{L^{* k}\right\}_{k=1}^{K}$, this estimated displacement field by itself does not necessarily minimize any formal registration energy.

\subsection{Equivalence to the random walker (RW) registration method}

We next show that a finite difference solution (FD) of the variational formulation in (3) is equivalent to the continuous-valued Gaussian MRF energy minimization formu- 
lation referred as the random walker registration in [1]. For this, consider the EulerLagrange equations corresponding to the energy functional in equation (3) for each $k \in\{1,2, \ldots, K\}$ :

$$
\nabla_{L^{k}}\left(E_{D}+\beta E_{R}^{\text {diff }}\right)=\left(L^{k}-\lambda^{k}\right)-\beta \nabla\left(W \nabla L^{k}\right)=0 .
$$

Rearranging we get for each $k \in\{1,2, \ldots, K\}$ :

$$
L^{k}-\beta \nabla\left(W \nabla L^{k}\right)=\lambda^{k} .
$$

Now, consider a uniform discretization $\mathcal{G}=\left(\left\{\mathbf{x}_{i}\right\}_{i=1}^{R}, \mathcal{N}\right)$ of the template image domain $\Omega_{T}$, where $\left\{\mathbf{x}_{i}\right\}_{i=1}^{R}$ is the set of pixels (voxels) and $\mathcal{N}$ denotes neighborhoods. Further, choose an image dependent stiffness field $\left.W(\mathbf{x})=\exp \left(-\gamma\left(\operatorname{diag}\left(\nabla I_{T}\right)\right)^{2}\right)\right)$. Writing the FDM approximation of the equations in (6) we obtain the following set of equations for each $k \in\{1,2, \ldots, K\}$ :

$$
\left(\operatorname{Id}_{N}+\beta \triangle_{\mathcal{G}}\right) \mathbf{L}^{k}=\boldsymbol{\lambda}^{k}
$$

Here, $\operatorname{Id}_{N}$ is a $N \times N$ identity matrix and $\triangle_{\mathcal{G}}$ is the discrete Laplacian operator defined on the uniform mesh $\mathcal{G}$ as:

$$
\left(\triangle_{\mathcal{G}}\right)_{i j}=\left\{\begin{array}{cl}
\sum_{r \in \mathcal{N}(i)} w_{i r} & \text { if } i=j \\
-w_{i j} & \text { if } j \in \mathcal{N}(i) \\
0 & \text { otherwise }
\end{array}\right.
$$

where $w_{i j}=\exp \left(-\gamma\left(I_{T}\left(\mathbf{x}_{i}\right)-I_{T}\left(\mathbf{x}_{j}\right)^{2}\right)\right)$. It is easy to observe that the FD discretization on the uniform grid $\mathcal{G}$ of the Euler-Lagrange equations in (5) corresponds to the set of linear equations in (7) that arise from the minimization of the continuous-valued MRF based discrete registration problem in [1].

\subsection{FEM-based solution for variational discrete deformable registration}

A more efficient solution of the proposed variational formulation for discrete registration (3) can be obtained using the FEM method. Consider a non-uniform discretization $\mathcal{M}=\left(\left\{P_{n}\right\}_{n=1}^{N}, \mathcal{T}\right)$ of the domain $\Omega_{T}$, where $\left\{P_{n}\right\}_{n=1}^{N}$ denotes the nodes of the mesh and $\mathcal{T}$ is the set of elements (triangles in $2 \mathrm{D}$, tetrahedra in 3D) (see Figure 1). Then using nodal basis functions $\left\{\phi_{n}\right\}_{n=1}^{N}$ we can discretize the probability maps as:

$$
\mathbf{L}(\mathbf{x})=\sum_{n=1}^{N} \mathbf{L}_{n} \phi_{n}(\mathbf{x} ; \mathcal{M}) \quad \forall \mathbf{x} \in \Omega_{T},
$$

where $\mathbf{L}_{n}=\mathbf{L}\left(P_{n}\right)$ which represents the value of the probability map at the node $P_{n}$ of the mesh $\mathcal{M}$. The task is now to find the nodal probabilities $\Theta=\left[\mathbf{L}_{n}\right]_{n=1}^{N}$. The FEM 
approximation of the energy in (3) can be written as:

$$
\begin{gathered}
\boldsymbol{\Theta}^{*}=\underset{\boldsymbol{\Theta} \in \mathbb{R}^{N K}}{\operatorname{argmin}} E_{D}\left(\boldsymbol{\Theta} ; I_{T}, I_{S}, \mathcal{D}\right)+\beta E_{R}^{\text {diff }}(\boldsymbol{\Theta}) \\
\text { with } \quad E_{D}\left(\boldsymbol{\Theta} ; I_{T}, I_{S}, \mathcal{D}\right)=\int_{\Omega_{T}}\left\|\sum_{n=1}^{N} \mathbf{L}_{n} \phi_{n}(\mathbf{x})-\boldsymbol{\lambda}(\mathbf{x} ; \mathcal{D})\right\|^{2} d \mathbf{x} \\
E_{R}^{\text {diff }}(\boldsymbol{\Theta})=\sum_{k=1}^{K} \sum_{m, n=1}^{N} L_{n}^{k} L_{m}^{k} \int_{\Omega_{T}} \nabla \phi_{n}^{\mathrm{T}}(\mathbf{x}) W(\mathbf{x}) \nabla \phi_{m}(\mathbf{x}) d \mathbf{x}
\end{gathered}
$$

where $\mathbf{L}_{n}=\left[L_{n}^{k}\right]_{k, n=1}^{K, N}$.

Setting the gradient of (10) to zero w.r.t each $\left\{\mathbf{L}_{n}\right\}_{n=1}^{N}$ and by re-writing $\boldsymbol{\Theta}=$ $\left[\boldsymbol{\Theta}^{k}\right]_{k=1}^{K}$, where $\boldsymbol{\Theta}^{k}=\left[L_{m}^{k}\right]_{m=1}^{N}$ is as a $N \times 1$ vector of nodal probabilities, we obtain the following system of linear equations for each $k \in\{1,2, \ldots, K\}$ :

$$
\mathbf{S} \Theta^{k}-\mathbf{F}^{k}=0
$$

where $\mathbf{S}=\left[S_{m, n}\right]_{m, n=1}^{N}$ is a $N \times N$ matrix and $\mathbf{F}^{k}=\left[F_{m}^{k}\right]_{m=1}^{N}$ is a $N \times 1$ vector defined as:

$$
\begin{aligned}
S_{m, n} & =\int_{\Omega_{T}} \phi_{m}(\mathbf{x}) \phi_{n}(\mathbf{x}) d \mathbf{x}+\beta \int_{\Omega_{T}} \nabla \phi_{m}^{\mathrm{T}}(\mathbf{x}) W(\mathbf{x}) \nabla \phi_{n}(\mathbf{x}) d \mathbf{x} \\
F_{m}^{k} & =\int_{\Omega_{T}} \lambda^{k}\left(\mathbf{x} ; \mathbf{d}^{k}\right) \phi_{n}(\mathbf{x}) d \mathbf{x} .
\end{aligned}
$$

The system of independent $N \times K$ linear equations in (11) is solved to obtain the optimal nodal probabilities $\boldsymbol{\Theta}^{*}=\left[\mathbf{L}_{m}^{*}\right]_{m=1}^{N}=\left[L_{m}^{* k}\right]_{k, m=1}^{K, N}$. Now, the optimal probabilities $\mathbf{L}^{*}(\mathbf{x})=\left[L^{* k}(\mathbf{x})\right]_{k=1}^{K}$ at any spatial location $\mathbf{x} \in \Omega_{T}$ can found through interpolation of the nodal probabilities $\left[\mathbf{L}_{m}^{*}\right]_{m=1}^{N}$ based on the finite element approximation in equation (9). Then, the estimated displacement field $\mathbf{U}(\mathbf{x})$ at $\mathbf{x} \in \Omega_{T}$ is obtained by choosing the displacement value $\mathbf{d}^{k}$ with the highest optimal probability at that spatial location, i.e., $\mathbf{U}(\mathbf{x})=\mathbf{d}^{k}$ where $k=\underset{r \in\{1,2, \ldots, K\}}{\operatorname{argmax}} L^{* r}(\mathbf{x})$.

\section{Experiments}

In this section, we evaluate our proposed FEM-based variational discrete deformable registration method on synthetic and real medical images. We implemented our method in a multi-resolution framework with 4 levels. The range of displacements used in each of the 4 levels are as follows: $[0, \pm 0.125, \ldots, \pm 0.5]^{\nu},[0, \pm 0.25, \ldots, \pm 1.0]^{\nu},[0, \pm 0.5$ $, \ldots, \pm 1.5]^{\nu},[0, \pm 1.0]^{\nu}$, where $\nu=2$ or 3 . We chose the Perona-Malik [16] diffusivity $W(\mathbf{x})=1 /\left(1+\exp \left(\left\|\nabla I_{T}\right\|^{2} / \gamma\right)\right)$ with $\gamma=0.05$ and set $\alpha=1.0$ in the experiments below. An image-adaptive meshing strategy proposed by Yang et al. [17] was used to generate the non-uniform FEM mesh. We coded our method in MATLAB using the MEX facility. We ran all experiments on a Intel i7 3.60 GHz machine with 32GB RAM. 


\subsection{Comparison with random walker (RW), DROP and demons registration}

In Figure 1, we show the performance of our FEM-based discrete registration method in comparison to the random walker (RW) [1], DROP [13] discrete registration methods and also the continuous diffusion-based demons [7] registration method on synthetic 2D brain MRI images with a known ground truth displacement field. The RW, DROP and demons methods all use a uniform mesh, while our FEM-based discrete registration method employs a non-uniform mesh well adapted to the image features. The number of DOFs associated with a mesh is given as twice the number of nodes in the mesh. Further, the computational times reported for the various algorithms correspond to the time taken by their respective optimization steps. In the case of our proposed method the optimization step involves solving the equation system in (11). It can be clearly seen that all the four registration methods successfully recover the displacement field between the two images. But, our FEM-based discrete registration method achieves this with a considerably lower computational effort $(\sim 30$ times lower DOFs and $>60$ times faster) when compared to the other three registration methods.

\subsection{Validation on the CUMC12 MRI database}

The CUMC12 dataset [14] consists of 3D brain MRI scans from 12 subjects taken at $256 \times 256 \times 124$ resolution with a $0.86 \mathrm{~mm} \times 0.86 \mathrm{~mm} \times 1.5 \mathrm{~mm}$ voxel spacing. Manual segmentations of 128 anatomical regions were provided for each of these images. We performed $12 \times 12-12=132$ pair-wise registrations between the images using our proposed FEM-based discrete registration method. The overall computational time for each registration is about 15.5 minutes of which 4.5 minutes are taken for the optimization step (solving equation system in (11)). For evaluating registration accuracy, anatomical labels on the source image were mapped to the template using the estimated displacement field. The Jaccard overlap measure was computed between the warped source labels and the template labels. In Figure 2, we report the Jaccard score obtained by our proposed method averaged over 128 anatomical regions and 132 pair-wise registrations. Klein et al. [18] reported average Jaccard scores obtained by 14 popular deformable registration methods on the CUMC12 dataset. We can see that our methods ranks in the top 5 among these methods.

\section{Conclusion}

We developed a computationally efficient FEM-based discrete deformable registration method using the squared differences similarity measure and diffusion-based regularization. A salient aspect of our method was the use of an image-adaptive discretization of the problem domain. This resulted in our method being multiple orders of magnitude faster than the existing registration methods that are implemented using uniform meshes. A limitation of our method is that the estimated displacement field is not diffeomorphic. This is because the displacement field is not explicitly regularized and the regularization is only implicit through the smoothing of prior probability maps. In future, we want to explore the use of an additional explicit regularization step that would yield diffeomorphic displacement fields. Further, we intend to extend our method for multi-modality registration using more complex similarity measures. 


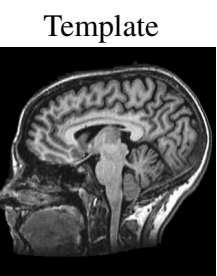

Source

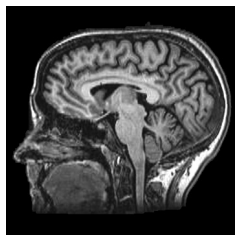

Ground truth

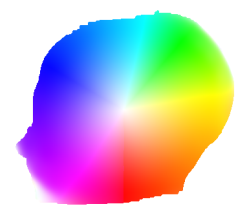

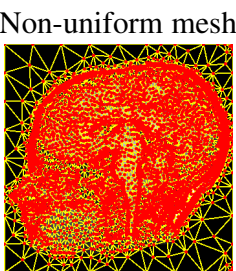

FEM discrete
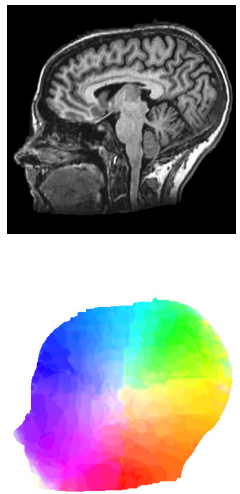

Color encoding

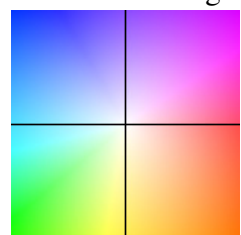

RW [1]

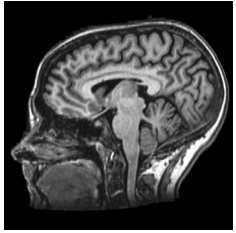

Demons [7]
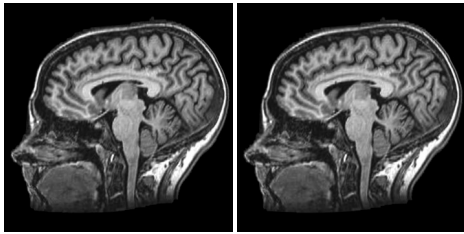
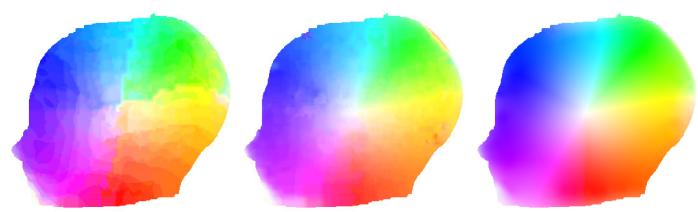

\begin{tabular}{|c|c|c|c|c|}
\hline Method & AAE & SSDE & DOF & Time(sec) \\
\hline Source (before registration) & - & 4866.67 & - & - \\
\hline FEM discrete (proposed) & $\mathbf{4 . 0 6}^{\circ}$ & $\mathbf{1 0 2 . 7 3}$ & $\mathbf{1 1 2 6 4}$ & $\mathbf{0 . 0 2}$ \\
\hline Random walker (RW) [1] & $4.72^{\circ}$ & 142.96 & 331588 & 1.27 \\
\hline DROP [13] & $2.48^{\circ}$ & 11.63 & 331062 & 54.74 \\
\hline Demons [7] & $1.93^{\circ}$ & 31.35 & 331588 & 122.20 \\
\hline
\end{tabular}

Fig. 1: Results on synthetic 2D MRI data. Average angular error (AAE), sum of squared differences error (SSDE) and degrees of freedom (DOF) are shown along with the computational times (we set $\beta=50$ in our proposed method).

\section{References}

1. Cobzas, D., Sen, A.: Random walks for deformable image registration. MICCAI (2011) 557-565 1, 2, 3, 4, 6, 7

2. Sotiras, A., Paragios, N., et al.: Deformable image registration: A survey. INRIA Research report (2012) 1

3. Broit, C.: Optimal registration of deformed images. PhD thesis, Univ. of Penn. (1981) 1

4. Modersitzki, J.: Numerical methods for image registration. Oxford Univ. Press (2004) 1

5. Fischer, B., Modersitzki, J.: Curvature based image registration. Journal of Mathematical Imaging and Vision 18(1) (2003) 81-85 1

6. Pennec, X., Cachier, P., Ayache, N.: Understanding the demons algorithm: 3d non-rigid registration by gradient descent. In: MICCAI. (1999) 597-605 1

7. Stefanescu, R., Pennec, X., Ayache, N.: Grid powered nonlinear image registration with locally adaptive regularization. Medical Image Analysis 8(3) (2004) 325-342 1, 6, 7 


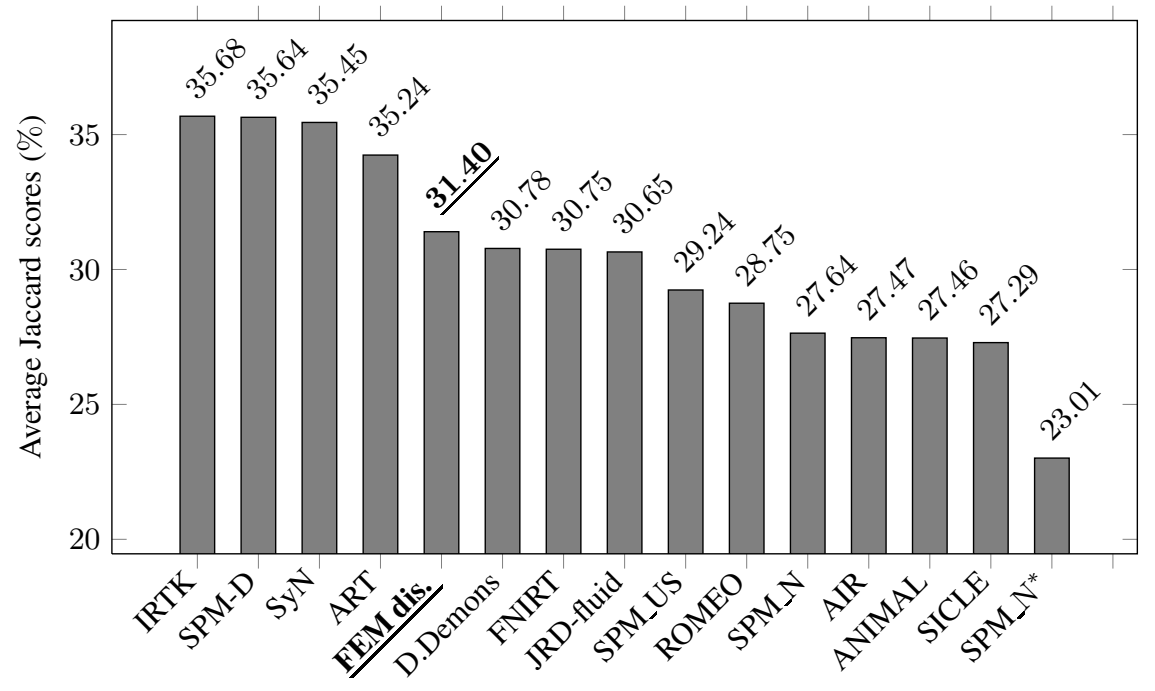

Fig. 2: Results on the CUMC12 MRI brain database (we set $\beta=200$ in our proposed method).

8. Cahill, N., Noble, J., Hawkes, D.: Demons algorithms for fluid and curvature registration. In: ISBI. (2009) 730-733 1

9. Rohde, G., Aldroubi, A., Dawant, B.: The adaptive bases algorithm for intensity-based nonrigid image registration. Medical Imaging, IEEE Tran. on 22(11) (2003) 1470-1479 1

10. Rueckert, D., Sonoda, L., Hayes, C., Hill, D., Leach, M., Hawkes, D.: Nonrigid registration using free-form deformations: application to breast mr images. Medical Imaging, IEEE Tran. on 18(8) (1999) 712-721 1

11. Jordan, P., Socrate, S., Zickler, T., Howe, R.: Constitutive modeling of porcine liver in indentation using $3 \mathrm{~d}$ ultrasound imaging. Journal of the mechanical behavior of biomedical materials 2(2) (2009) 192-201 1

12. Tang, T., Chung, A.: Non-rigid image registration using graph-cuts. In: MICCAI. (2007) 916-924 2

13. Glocker, B., Komodakis, N., Tziritas, G., et al.: Dense image registration through mrfs and efficient linear programming. Medical Image Analysis 12(6) (2008) 731-741 2, 6, 7

14. : Columbia univ. medical centre mri dataset. Www.mindboggle.info/papers/ evaluation_NeuroImage2009/data/CUMC12.tar.gz Accessed: 1/3/2013. 2, 6

15. Grady, L.: Multilabel random walker image segmentation using prior models. In: CVPR. Volume 1. (2005) 763-770 3

16. Perona, P., Malik, J.: Scale-space and edge detection using anisotropic diffusion. Pattern Analysis and Machine Intelligence, IEEE Tran. on 12(7) (1990) 629-639 5

17. Yang, Y., Wernick, N., Brankov, G.: A fast approach for accurate context-adaptive mesh generation. IEEE transactions on image processing 12(8) (2003) 866-881 5

18. Klein, A., Andersson, J., Ardekani, B., Ashburner, J., Avants, B., Chiang, M., Christensen, G., Collins, D., Gee, J., Hellier, P., et al.: Evaluation of 14 nonlinear deformation algorithms applied to human brain mri registration. Neuroimage 46(3) (2009) 786-802 6 